CASE REPORT

\title{
Masquerade: a malignant arrhythmia masquerading as benign
}

\section{R Nandakumar, J C Patel, P Broadhurst}

Heart 2004;90:e10 (http://www.heartjnl.com/cgi/content/full/90/2/e10) doi:10.1136/hrt.2003.24067

Pseudoatrial flutter has been reported to have benign causes, but this is the first report of a malignant arrhythmia presenting as a benign arrhythmia. An 82 year old patient presented with ventricular tachycardia and electrical artefact appearing as atrial flutter. In this case, comparing the morphology of the QRS complexes in the rhythm strip with those in lead II showed the arrhythmia to be ventricular in origin and points to the importance of comparing all leads of the ECG before arriving at a diagnosis.

A n 82 year old man presented with chest pain lasting 90 minutes, breathlessness, and sweating, but without palpitations. He had a history of myocardial infarction and was known to have had a transient episode of atrial fibrillation after myocardial infarction.

Figure 1 shows the ECG recorded by the attending paramedic, which was interpreted as showing atrial flutter with variable block. The arrhythmia was unresponsive to Valsalva manoeuvre, carotid sinus massage, or intravenous lidocaine. Further examination of other leads (fig 2) showed the true diagnosis to be ventricular tachycardia.

The arrhythmia was unresponsive to Valsalva manoeuvre, carotid sinus massage, or intravenous lidocaine.

Close inspection of the rhythm strip and lead II shows identical QRS morphology in both leads. However, the rhythm strip is interspersed with electrical artefacts, which was interpreted as QRS complexes. This artefact was interpreted as pacemaker spikes by the defibrillator in the ambulance.

\section{DISCUSSION}

Pseudoatrial flutter is the simulation of atrial flutter by various artefacts such as a broken wire in an extremity electrode, ${ }^{1}$ Parkinson's disease, ${ }^{2-4}$ myoclonic contractions in the pectoralis major due to cold, ${ }^{5}$ setting up of wave motion in condensed water within oxygen tubing, ${ }^{6}$ diaphragmatic flutter or hiccups, loose connections, and improper lead placement. ${ }^{7}$

The cause of transient artefact in our patient is unclear but serves as a timely reminder to examine carefully all leads of the ECG before arriving at a diagnosis.

\section{Author's affiliations}

R Nandakumar, J C Patel, P Broadhurst, Aberdeen Royal Infirmary, Aberdeen, UK

Correspondence to: Dr R Nandakumar, Department of Cardiology, Aberdeen Royal Infirmary, Aberdeen, UK; nandakumar.ramasami@arh.grampian.scot.nhs.uk

Accepted 2 October 2003

\section{REFERENCES}

1 Rubenfire M, Rosenzweig S. Electrocardiographic artifacts simulating atrial flutter. JAMA 1972;220:1130.

2 Pallis CA, Calne DB. Parkinsonism and cardiac arrhythmias. Lancet 1970;ii:1313.

3 Young AC, Downie AW. Parkinsonism and cardiac arrhythmias. Lancet 1971;i:139-140.

4 Saint-Pierre A. ECG artifacts simulating atrial flutter. JAMA 1973;224:1534.

5 Borrello $G$. Electrocardiographic artifacts simulating atrial flutter. JAMA 1973:223:439.

6 Vannelli A, Fuller HD. An unusual artifactual cause of apparent atrial flutter. Am Heart J 1989;117:202-4.

7 Castellano A, Myerburg AJ. The resting electrocardiogram, 6th edn. New York: McGraw Hill International, 1986:225-7.

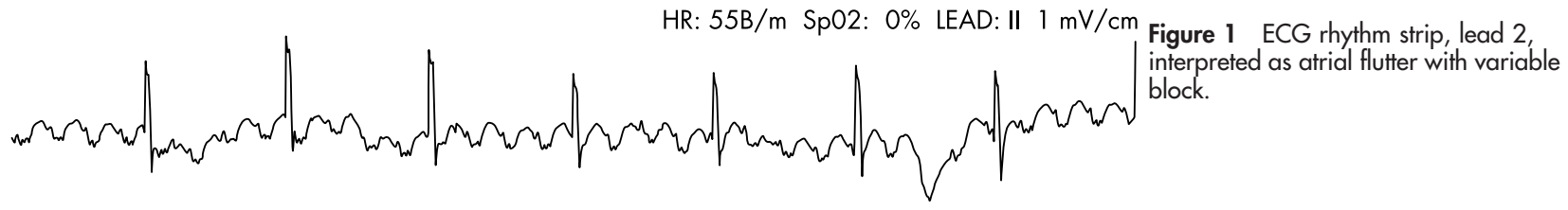

HR: 228B/m LEAD: II $1 \mathrm{mV} / \mathrm{cm}$

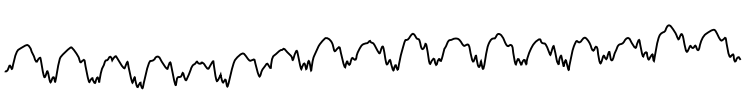

HR: 228B/m LEAD: III $1 \mathrm{mV} / \mathrm{cm}$ Figure 2 ECG leads 2, 3, and 1, leading to the true diagnosis of ventricular tachycardia.

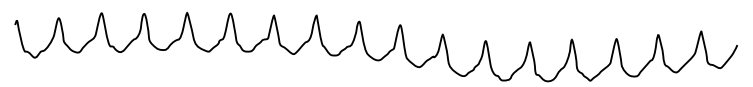

